\title{
Qiweibaizhu Decoction Treats Diarrheal Juvenile Rats by Modulating the Gut Microbiota, Short-Chain Fatty Acids, and the Mucus Barrier
}

\author{
Shaodan Sun $\left(\mathbb{D},{ }^{1,2}\right.$ Yang Yang $\left(\mathbb{D},{ }^{3,4}\right.$ Xiaojie Lin, ${ }^{5}$ Peiwen Chen, ${ }^{6}$ Liyan Ye, ${ }^{6}$ Liying Zeng, ${ }^{6}$ \\ Qina Ye, ${ }^{7}$ Xiangna Yang, ${ }^{7}$ Jingtu Ceng, ${ }^{8}$ Jiayi Shan, ${ }^{6}$ Li Xie, ${ }^{6}$ Meirong Jiang, ${ }^{6}$ Fei Luo $\mathbb{D},{ }^{6}$ \\ and Xiaogang Chen (iD) ${ }^{6,9}$ \\ ${ }^{1}$ The Second Clinical College of Guangzhou University of Chinese Medicine, Guangzhou University of Chinese Medicine, \\ Guangzhou 510120, China \\ ${ }^{2}$ Lingnan Medical Research Center, Guangzhou University of Chinese Medicine, Guangzhou 510407, China \\ ${ }^{3}$ Affiliated Jiangmen Traditional Chinese Medicine Hospital of Ji'nan University, Ji'nan University, Jiangmen 529000, China \\ ${ }^{4}$ Integrated Chinese and Western Medicine Postdoctoral Research Station, Jinan University, Guangzhou 510632, China \\ ${ }^{5}$ Guangdong Provincial Hospital of Traditional Chinese Medicine, Guangzhou 510120, China \\ ${ }^{6}$ Department of Pediatrics, The First Affiliated Hospital of Guangzhou University of Chinese Medicine, Guangzhou 510405, China \\ ${ }^{7}$ Guangzhou Institute of Pediatrics, Guangzhou Women and Children's Medical Center, Guangzhou 510407, China \\ ${ }^{8}$ Tongde Hospital of Zhejiang Province, Hangzhou 310012, China \\ ${ }^{9}$ Guangzhou University of Chinese Medicine, Guangzhou 510405, China
}

Correspondence should be addressed to Fei Luo; luofeigz@126.com and Xiaogang Chen; wyzfz@163.com

Received 14 August 2020; Revised 18 November 2020; Accepted 22 December 2020; Published 18 January 2021

Academic Editor: Wan Mohd Aizat

Copyright (C) 2021 Shaodan Sun et al. This is an open access article distributed under the Creative Commons Attribution License, which permits unrestricted use, distribution, and reproduction in any medium, provided the original work is properly cited.

Background. Qiweibaizhu decoction (QBD), a classic Chinese herbal formula, has been widely used for treating diarrhea in infants and children with spleen deficiency syndrome for centuries, but its mechanism of action remains unclear. The gut microbiota, short-chain fatty acids (SCFAs), and intestinal mucus are closely associated with diarrhea. Methods. In this study, the composition of the gut microbiota in diarrheal rats was analyzed by $16 \mathrm{~S}$ rDNA amplicon sequencing. The concentrations of colon SCFAs were determined using gas chromatography-mass spectrometry (GC-MS). The expression of mucin 2 (MUC2) in the colon was detected by immunofluorescence. Results. Diarrhea significantly changed the diversity and structure of the gut microbiota and disrupted the mucus barrier in juvenile rats. QBD did not significantly change the diversity and structure of the intestinal flora, but it enhanced the increasing tendencies of Verrucomicrobia and Akkermansia and decreased the abundance of Turicibacter $(P=0.037)$ and Flavonifractor $(P=0.043)$. QBD tends to repair the mucus layer and promote MUC2 expression in juvenile rats with diarrhea. Moreover, $S$. boulardii significantly increased the abundance of Parasutterella $(P=0.043)$. In addition, QBD treatment tends to increase the propionic acid concentration during diarrhea, but its levels of acetic acid, propionic acid, butyric acid, and total SCFAs were lower than those in the S. boulardii group. Conclusion. S. boulardii significantly increased the abundance of Parasutterella, leading to increased production of acetic acid, propionic acid, and butyric acid, consequently leading to alleviation of diarrhea. In comparison, QBD affected diarrhea via regulation of the intestinal flora, especially by increasing the abundance of Verrucomicrobia and Akkermansia, resulting in mucus barrier repair, protection of the intestines, and treatment of diarrhea.

\section{Introduction}

Diarrhea was the eighth leading cause of death among people of all ages and the fifth leading cause of death among children under 5 years of age in 2016, especially in South Asia and sub-Saharan Africa [1, 2]. Osmotic diarrhea is a common disease in humans caused by food intolerances, malabsorption, and the widespread use of laxatives [3]. 
Polyethylene glycol (PEG) is a commonly used digestive remedy to induce osmotic diarrhea. Osmotic diarrhea can significantly change the microbial community structure, which is similar to changes observed in other gastrointestinal diseases, including inflammatory bowel disease (IBD) [4].

Diarrheal disease is associated with dysbiosis of the gut microbiota in children [5]. The Global Enteric Multicenter Study found that moderate-to-severe diarrhea reduced bacterial diversity and altered microbiota composition in children [6]. Children with multiple episodes of diarrhea in early life may suffer impairment in gut microbiota development that could result in persistent diarrhea, malnutrition, and immune system diseases. Pre- and/or probiotics can modify the microbiota and improve the outcomes $[7,8]$.

Short-chain fatty acids (SCFAs), mainly produced by various gut microflora in the colon, are fatty acids with carbon chains containing less than 8 carbon atoms, mainly including acetic acid, propionic acid, and butyric acid [9]. SCFAs are an important energy for intestinal cells and are key signaling molecules for maintaining intestinal health [10]. SCFAs are absorbed by colonic epithelial cells and stimulate $\mathrm{Na}$-dependent water and electrolyte absorption to alleviate diarrhea symptoms [11]. Specifically, sodium butyrate can also prevent diarrhea by increasing the passive absorption of water by the colon and affecting the intestinal microflora [12].

Mucin is a large, highly glycosylated protein that is important for protecting the lumen of the gastrointestinal tract [13].Recently, a large number of studies have shown that the mucus barrier and its most important protein, mucin 2 (MUC2), protect the intestines and prevent diarrhea. Tropini et al. confirmed that diarrhea is closely related to the gut microbiota and the intestinal mucus barrier. Diarrhea significantly changes the gut microbiota and makes the mucus layer thin or absent [3]. Changes in the composition of the intestinal flora and the balance of the environment in the intestine can lead to a decrease in MUC2 secretion [14].

Probiotics in the pediatric population have been widely studied in the prevention and treatment of diarrheal diseases [15]. S. boulardii is a nonpathogenic probiotic yeast that is tolerated with gastric acid and antibiotics [16]. S. boulardii has been reported to reduce signs of diarrhea related to or caused by various factors in animals and humans $[17,18]$. The mechanisms by which $S$. boulardii treats diarrhea are as follows: (1) S. boulardii can inhibit the growth and invasion of pathogens [19]. (2) S. boulardii can inhibit host cell adherence that interferes with bacterial colonization [20]. (3) S. boulardii has an antisecretory effect [21]. (4) Importantly, $S$. boulardii was reported to reduce bacterial gut translocation and improve intestinal barrier function in animal models [22]. S. boulardii can affect the composition and release of intestinal mucin, enhance the mucin barrier, and reduce the penetration of SN-38 into epithelial cells, thereby reducing mucosal damage [23]. At present, the mechanism of action of $S$. boulardii is not yet fully understood, and the optimal dose of its action remains to be explored.

In traditional Chinese medicine (TCM), combination therapies (also called formulae) have been used for
2,500 years. Formulae are a combination of several types of medicinal herbs or minerals, which aim to enhance therapeutic efficacy and reduce adverse effects [24]. The Qiweibaizhu decoction (QBD), consisting of Guanghuoxiang (Pogostemon cablin (Blanco) Benth.), Gegeng (Radix Puerariae), Baizhu (Atractylodes macrocephala Koidz.), Rensheng (Panax ginseng C. A. Mey.), Fuling (Poria $\operatorname{cocos}($ Schw.) Wolf.), Muxiang (Aucklandiae Radix), and Gancao (Licorice), has been used to treat the diarrhea associated with spleen deficiency syndrome since the Song Dynasty (960-1127 AD). The term "spleen" in traditional Chinese medicine (TCM) is different from that in Western medicine. Spleen deficiency syndrome is characterized by poor appetite, fullness, fatigue, pale tongue coating, weight loss, and loose stools. The commonly used treatment duration of $\mathrm{QBD}$ is 3-14 days. The commonly used dose range of QBD is $0.64-2.56 \mathrm{~g} / \mathrm{ml} ; 0.64 \mathrm{~g} / \mathrm{ml}$ is a low dose, $2.56 \mathrm{~g} / \mathrm{ml}$ is a high dose, and the most commonly used dose is $1.6 \mathrm{~g} / \mathrm{ml}$ [25-28]. In these safe ranges, no obvious side effects or adverse reactions have been reported [29-33]. In this experiment, the dose of QBD was $1.20 \mathrm{~g} / \mathrm{ml}$ for 10 days, which is a low dose compared with other experiments. Moreover, the seven traditional Chinese medicines in $\mathrm{QBD}$ are very safe traditional Chinese herbs, which can be eaten as food. In traditional Chinese medicine, these are called "drug homologous food."

A number of studies have confirmed that QBD can balance the gut microbiota by promoting the growth of beneficial bacteria and inhibiting the reproduction of harmful bacteria [34]. The therapeutic effect of QBD depends on its various effective chemical components, such as polysaccharides, saponins, proteins, daidzein, and flavonoids, which are the material basis for the therapeutic effects of QBD [35-40]. Another study found that QBD contains a small amount of prebiotic substances; these compounds can promote the growth of probiotics in the intestine and can promote the synthesis of a variety of proteins and vitamins in the intestine [41]. Moreover, Sun et al. found that QBD is effective for treating diarrhea in mice by regulating the imbalance of intestinal flora and promoting the repair of intestinal mucosal damage [32]. Zhang et al. showed that the four herbs in QBD can increase the expression of MUC2 to improve the state of spleen deficiency in rats [62].

Therefore, we hypothesized that QBD could regulate the structure of the gut microbiota and the levels of SCFA and MUC2 in the treatment of diarrhea. Specifically, we applied QBD to treat juvenile rats with diarrhea and compared the results with those obtained with $S$. boulardii to explore whether QBD treats diarrhea by modulating the gut microbiota and SCFA-producing bacteria to increase the concentrations of SCFAs or MUC2-producing bacteria to increase the expression of MUC2.

\section{Methods}

2.1. Identification of Bioactive Ingredients of $Q B D$. The Traditional Chinese Medicine System Pharmacology Database and Analysis Platform (TCMSP) (http://tcmspw.com/ tcmsp.php) is a systems pharmacology platform for Chinese 
medicine. By using the TCMSP, the chemical components of each Chinese medicine compound in QBD can be obtained, and the bioactive ingredients can be screened according to the absorption, distribution, metabolism, and excretion (ADME) information of the Chinese medicine components. The screening criteria are as follows: $\mathrm{OB}$ value (oral bioavailability) $\geq 30 \%, \mathrm{OB} \% \geq 60 \%$ for (Gancao) licorice, and DL value (drug-likeness) $\geq 0.18$ [42].

2.2. Preparation of $Q B D$. $\mathrm{QBD}$ was prepared by combining seven herbs in a ratio of $10: 10: 10: 10: 5: 4: 2$ by weight of Guanghuoxiang (Pogostemon cablin (Blanco) Benth.), Gegeng (Radix Puerariae), Baizhu (Atractylodes macrocephala Koidz.), Rensheng (Panax ginseng C. A. Mey.), Fuling (Poria $\operatorname{cocos}$ (Schw.) Wolf.), Muxiang (Aucklandiae Radix), and Gancao (Licorice). These herbs were obtained from Guangdong Provincial Hospital of Traditional Chinese Medicine. These herbal materials were soaked in 4 times the volume of cold water in a beaker for $30 \mathrm{~min}$ and decocted 2 times (45 min each time). Rensheng (Panax ginseng C. A. Mey.) was soaked and decocted separately. The above mixture was thus obtained and concentrated to a concentration of $1.20 \mathrm{~g}$ of the original medicinal material per milliliter of the medicinal liquid by using a rotary evaporator in an $80^{\circ} \mathrm{C}$ water bath. After the decoction had cooled, it was stored in a freezer at $-20^{\circ} \mathrm{C}$ until use.

2.3. S. boulardii Preparation. S. boulardii (Ultra-Levure ${ }^{\circledR}$ from Biocodex) was suspended in distilled water and administered orally. Each bag contained $765 \mathrm{mg}$ of powder and $250 \mathrm{mg}$ of bacterial powder; the number of viable bacteria per $1 \mathrm{~g}$ of powder should not be less than $1.3 \times 10^{9} \mathrm{CFU}$. A solution $\left(12 \times 10^{10} \mathrm{CFU} / \mathrm{kg}\right)$ was prepared with pure water.

2.4. Experimental Animals and Modeling. Forty-eight specific-pathogen-free male Sprague-Dawley rats (age, 5 weeks; weight, $142 \pm 8 \mathrm{~g}$ ) were purchased from the Guangdong Animal Experimental Center (license no. SCXK (Yue) 2018-0002). The rats were kept in standard cages at the Experimental Animal Center of the First Affiliated Hospital of Guangzhou University of Chinese Medicine (license no. SYXK (Yue) 2013-0092) under standard environmental conditions $\left(21-25^{\circ} \mathrm{C} ; 50 \%-60 \%\right.$ humidity; $12 \mathrm{~h}$ light/dark cycle). Diet and water were freely available throughout the experiment. All experiments involving animals were approved by the Animal Experimentation Ethics Committee of the First Affiliated Hospital of Guangzhou University of Chinese Medicine (approved application no. TCMF12019036) and were in compliance with the Guidelines for the Care and Use of Laboratory Animals published by the US National Institutes of Health. All efforts were made to minimize the suffering of the animals.

2.5. Experimental Processes. The rats were randomly assigned to 4 groups $(n=12)$ : control, model control, $S$. boulardii, and QBD groups. The control group did not receive any treatment; the model group, S. boulardii group, and QBD group were treated with polyethylene glycol (PEG) water (15\% PEG 3350 was added to the drinking water for the first three days, and 10\% PEG 3350 was added for the remaining six days) and were placed on a small platform in a water environment to stand for 6 hours for 9 days.

After the nine days and 12 hours of PEG induction, rats in the model, S. boulardii, and QBD groups were intragastrically given normal saline $(1 \mathrm{ml} / \mathrm{kg}), S$. boulardii $(1 \mathrm{ml} /$ $\mathrm{kg})$, and QBD ( $1 \mathrm{ml} / \mathrm{kg})$, respectively, once a day for 10 days. Defecation, body weight, diet, water, and general clinical conditions were monitored every day.

Colon contents were collected and immediately frozen with liquid nitrogen after sampling and stored at $-80^{\circ} \mathrm{C}$. Tissue samples from the colon were immediately fixed in methanol-Carnoy's solution (60\% dry methanol, $30 \%$ chloroform, and $10 \%$ glacial acetic acid).

2.6. Diarrhea Assessment. Stool consistency was classified according to the following visual grading scale as previously described [43]: (1) formed, stool maintains its shape, brown, score $=1$; (2) semiformed or soft, does not pour, yellow, score $=2$; and (3) liquid, pours more easily, yellow, score $=3$.

\subsection{Analysis of $16 S$ rDNA in the Colon Contents}

2.7.1. Sample Collection and DNA Extraction. The composition of the gut microbiota was detected by $16 \mathrm{~S}$ rDNA amplicon sequencing analysis. Total DNA was isolated from the colon content samples with the DNA Stool Mini Kit (Qiagen, Hilden, Germany) according to the manufacturer's protocols. DNA concentration and purity were determined by spectrophotometry (ND-2000 spectrophotometer, Thermo Fisher Science, Waltham, MA, USA). DNA integrity and concentration were assessed using 1\% agarose gel electrophoresis.

2.7.2. 16S rRNA Gene Amplification, Sequencing, and Illumina MiSeq. The V3-V4 region of the bacterial $16 \mathrm{~S} \mathrm{rDNA}$ was amplified by PCR $\left(95^{\circ} \mathrm{C}\right.$ for $5 \mathrm{~min} ; 25-28$ cycles of $98^{\circ} \mathrm{C}$ for $20 \mathrm{~s}, 58^{\circ} \mathrm{C}$ for $15 \mathrm{~s}$, and $72^{\circ} \mathrm{C}$ for $20 \mathrm{~s}$; and $72^{\circ} \mathrm{C}$ for $5 \mathrm{~min}$, followed by holding at $4^{\circ} \mathrm{C}$ ). The amplicon was detected using 2\% agarose gel electrophoresis and purified by the AxyPrep DNA Gel Extraction Kit (Axygen Biosciences, Union City, CA, USA). The amplified products were generated using the Illumina MiSeq system (Illumina, San Diego, CA, USA) with barcoded primers. The amplicons were sequenced (PE250) on an Illumina MiSeq platform (Shanghai Realbio Institute, Shanghai, China) to obtain $250 \mathrm{bp}$ paired-end reads.

2.7.3. Sequencing Data Analysis. Paired-end sequencing was performed, and *_1.fq and *_2.fq each correspond to a FASTQ file. Paired-end sequencing was performed on the Illumina platform. The reads were spliced based on the overlap between reads, and quality control of the spliced reads was performed to obtain clean reads. The optimized clean reads were obtained by Pandaseq, and an in-house 
program was used to process the spliced reads as follows to obtain clean reads: (1) reads with an average quality value of less than 20 were removed; (2) reads with more than $3 \mathrm{~N}$ bases were removed; and (3) reads not in the length range of 220 500 nt were removed. The operational taxonomic units (OTUs) for species classification were obtained by using usearch for clustering with 0.97 similarity, and chimeric filtering was performed on the clustered sequences.

\subsection{Determination of SCFAs in the Colon Contents}

2.8.1. Sample Preparation. Colon contents $(50 \mathrm{mg})$ were weighed. Saturated sodium chloride solution $(400 \mu \mathrm{L})$ and saturated sodium chloride solution with hydrochloric acid $(50 \mu \mathrm{L}, 3 \mathrm{mmol})$ were added, and the combination was mixed by ultrasonication for 1 hour at low temperature. Cold ether $(500 \mu \mathrm{L})$ was then added to the combination. The mixture was then centrifuged at $12000 \mathrm{r} / \mathrm{min}$ and $4^{\circ} \mathrm{C}$ for $10 \mathrm{~min}$. The supernatant was removed and collected into a tube containing $0.1 \mathrm{~g}$ of anhydrous sodium sulfate and vortexed for $3 \mathrm{~min}$. Finally, the supernatant was obtained by centrifugation $\left(4500 \mathrm{r} / \mathrm{min}\right.$ and $4^{\circ} \mathrm{C}$ for $\left.5 \mathrm{~min}\right)$ for determination of the SCFAs by gas chromatography-mass spectrometry (GC-MS).

2.8.2. Instrumental Conditions. The instrument used in this experiment was a GC-MS Agilent 7890B gas chromatograph and an Agilent 5977A MSD mass spectrometer in series for detection in selected ion monitoring (SIM) mode. MSD ChemStation software was used for data processing to calculate the absolute content of the target compound in the sample. (1) The required gas chromatographic parameters were as follows: chromatographic column: Agilent HPFFAP $(25 \mathrm{~m}+0.32 \mu \mathrm{m}+0.50 \mathrm{~mm})$; injection volume: $2 \mu \mathrm{L}$; split ratio: $20: 1$; injection port: $250^{\circ} \mathrm{C}$; flow rate: $1.5 \mathrm{~mL} / \mathrm{min}$; heating program: the oven program had an initial temperature of $100^{\circ} \mathrm{C}$, which was increased to $150^{\circ} \mathrm{C}$ at a rate of $5^{\circ} \mathrm{C} / \mathrm{min}$, and maintained at $230^{\circ} \mathrm{C}$ for $2 \mathrm{~min}$. (2) The mass spectrometry parameters were as follows: ion source: $\mathrm{EI}+$; ion source temperature: $280^{\circ} \mathrm{C}$; transmission line temperature: $250^{\circ} \mathrm{C}$; solvent delay: $3.5 \mathrm{~min}$; scanning range: scan $35-200 \mathrm{~m} / \mathrm{z}$. The SIM mode was chosen to determine the ion mass of each SCFA, including $\mathrm{m} / \mathrm{z}$ values of $41,43,45,57,60$, $73,74,87$, and 88 .

2.9. Alcian Blue Staining. Tissue samples from the colon were immediately fixed in methanol-Carnoy's solution $(60 \%$ dry methanol, $30 \%$ chloroform, and $10 \%$ glacial acetic acid) and processed in paraffin as previously described $[44,45]$. Sections were washed in xylene I for $20 \mathrm{~min}$, xylene II for $20 \mathrm{~min}$, anhydrous ethanol I for $5 \mathrm{~min}$, anhydrous ethanol II for $5 \mathrm{~min}, 75 \%$ alcohol for $5 \mathrm{~min}$, and tap water for $5 \mathrm{~min}$. Sections were stained with Alcian blue dye for $15 \mathrm{~min}$ and then washed with tap water. Finally, sections were dehydrated with anhydrous ethanol I ( $5 \mathrm{~min}$ ), anhydrous ethanol II (5 min), anhydrous ethanol III (5 min), xylene I (5 min), and xylene II (5 min).
2.10. Immunofluorescence. Tissue samples from the colon were fixed and processed as described for Alcian blue staining. Sections were washed in xylene I for $15 \mathrm{~min}$, xylene II for $15 \mathrm{~min}$, anhydrous ethanol I for $5 \mathrm{~min}$, anhydrous ethanol II for $5 \mathrm{~min}, 85 \%$ alcohol for $5 \mathrm{~min}, 75 \%$ alcohol for $5 \mathrm{~min}$, and distilled water for $5 \mathrm{~min}$. Sections were immersed in citric acid antigen retrieval solution and boiled in a microwave oven $(300 \mathrm{~W})$ for 5 min twice. Then, the sections were soaked in hot solution for $20 \mathrm{~min}$. Sections were washed in PBS 3 times (each time for $5 \mathrm{~min}$ ) and marked with a PAP (liquid blocker) pen. BSA was added, and the samples were incubated in the dark in a humid chamber for $30 \mathrm{~min}$ at room temperature. A specific antibody for MUC2 (Servicebio, diluted 1:200 in blocking solution) was added to the slide, which was then incubated in darkness for 4-24 hours at $4^{\circ} \mathrm{C}$. The slides were washed three times in PBS. The secondary antibody (Servicebio, diluted 1:200 in blocking solution) was added to the sample, which was then incubated in the dark at room temperature for $50 \mathrm{~min}$. After incubation with the secondary antibody, slides were washed three times in PBS and dried. DAPI (Servicebio) was then added to the slides for incubation in the dark at room temperature for 10 min. Finally, the slides were washed three times in PBS and mounted with Antifade Mounting Medium (Servicebio). Slides were stored at $4^{\circ} \mathrm{C}$ in the dark until imaging.

\section{Data Analysis}

Statistical analysis was performed with STATA 12.0 and SPSS 24.0 software. If the measured data fit a normal distribution, the values are presented as the mean \pm standard deviation; for data that did not fit a normal distribution, the median (interquartile range) was used. Significant differences between groups were assessed by analysis of variance, the Kruskal-Wallis test, and the Mann-Whitney test. $P<0.05$ was considered statistically significant.

\section{Results}

4.1. Identification of the Bioactive Compounds in $Q B D$. From the seven active components of QBD, Guanghuoxiang (Pogostemon cablin (Blanco) Benth.), Gegeng (Radix Puerariae), Baizhu (Atractylodes macrocephala Koidz.), Rensheng (Panax ginseng C. A. Mey.), Fuling (Poria $\operatorname{cocos}$ (Schw.) Wolf.), Muxiang (Aucklandiae Radix), and Gancao (Licorice), 777 compounds were obtained from the Traditional Chinese Medicine Systems Pharmacology (TCMSP) database. Fortysix active chemical components of QBD were obtained with an $\mathrm{OB} \% \geq 30 \%$ (Glycyrrhiza is $\mathrm{OB} \% \geq 60 \%$ ) and a $\mathrm{DL} \geq 0.18$, according to the screening standards. The properties of the compounds are shown in Supplementary Table 1.

4.2. Efficacy of $Q B D$ in Alleviating Diarrhea. Diarrhea was not observed in any of the rats in the control group. From 1 day after the ingestion of PEG, watery stools or diarrhea began to appear in the model group, S. boulardii group, and QBD group. Notably, rats in the model group exhibited obvious diarrhea, as determined by measuring fecal consistency and weight loss. 
On the first day of the experiment, the mean weights of rats in all groups were similar. During the study period, weight loss was not detected in the control group, but the weight of the rats in the remaining three groups decreased. From the second day of PEG treatment to the last day of the experiment, the mean weights of the model group, $S$. boulardii group, and QBD group were lower than that of the control group $(P<0.05)$. Pairwise comparisons between the model group and the $S$. boulardii and $\mathrm{QBD}$ groups showed that the $P$ value was greater than 0.05 , and there was no significant difference. However, the mean weights of the three groups were in the following order: QBD group $>S$. boulardii group $>$ model group (see Figure 1).

4.3. Effect of $Q B D$ on the Colon Mucus Barrier. Alcian blue staining revealed that, in the model group, the mucus layer was extremely thin or even absent, only a few goblet cells secreted new mucus from the base, and most of the goblet cells did not secrete new mucus compared to the control group. Compared to the control group, the thickness of the mucus layer in the model group was significantly thinner $(P=0.001)$.

In the $S$. boulardii group and $\mathrm{QBD}$ group, the mucus layer was incomplete but thicker and more continuous than that in the model group. Most goblet cells secreted new mucus from the base. Compared to the model group, the thickness of the mucus layer in the $\mathrm{QBD}$ group $(P=0.06)$ and the $S$. boulardii group $(P=0.22)$ was not statistically significant. However, the thickness of the mucus layer in the QBD group was not significantly different $(P=0.08)$ from that in the blank control group. The results showed that both the $S$. boulardii group and QBD group showed reversal of PEG-induced mucus thinning compared with the model group.

Immunofluorescence-stained sections revealed that the expression of MUC2 was extremely weak in the intestinal lumen and goblet cells compared to the control group. MUC2 had stronger fluorescence expression in the S. boulardii group and QBD group than in the model group, indicating that the layer thickness, the level of MUC2 expression, and the increase in MUC2 secretion by goblet cells were restored compared to those in the model group, consistent with the Alcian blue staining results (see Table 1 and Figure 2).

4.4. Effects of QBD Treatment on SCFAs in the Colon Contents. Seven SCFAs were detected in the colon contents via GCMS. The concentrations of total SCFAs, acetic acid, and propionic acid increased, and the propionic acid concentration $(P=0.047)$ reached statistical significance in the model group compared to the control group. However, the level of butyric acid $(P=0.02)$ in QBD group was significantly reduced compared to that in the control group. Although the difference was not statistically significant, the total levels of SCFAs, acetic acid, propionic acid, and butyric acid in the $S$. boulardii group were higher than those in the QBD group or the model group. These results showed that the effect of $S$. boulardii on SCFA production was better than that of QBD (see Figure 3).

4.5. Overall Structural Modulation of the Gut Microbiome during QBD Treatment. The structural changes in the rat gut microbiota after QBD treatment were detected by $16 \mathrm{~S}$ rDNA amplicon sequencing. A total of 1,420,548 reads (average of 35,514 sequences per sample) from 40 samples were delineated into 667 OTUs. The species accumulation curves showed an adequate sampling depth for all samples (see Figures 4(a) and 4(b)).

Alpha diversity indices (Chaol and Shannon) reflect community richness and uniformity. The highest community richness and uniformity were observed in the control group. Compared with the control group, the Chaol index and Shannon index were significantly lower in the model group. However, the differences in the Shannon index among the model, S. boulardii, and QBD groups were not significant (see Figures 4(c) and 4(d)).

Beta diversity analysis includes nonmetric multidimensional scaling (NMDS) and principal co-ordinates analysis (PCoA). The NMDS and PCoA results showed that the distance between the control group and the other three groups was large. In summary, PEG treatment reduced the species diversity and structure of the gut microbiota in rats, while S. boulardii and QBD treatment did not significantly change the species diversity and structure of the gut microbiota (see Figures 5(a) and 5(b)).

4.6. Key Phylotypes of the Gut Microbiota Modulated by the $Q B D$. The composition of the gut microbiota at different taxonomic levels was analyzed to determine which types of bacteria were affected by S. boulardii and QBD intake.

Eight phyla were found in all of the samples, among which the most abundant were Bacteroidetes, Firmicutes, Verrucomicrobia, and Proteobacteria. At the genus level, twenty-two genus-dominant genera were identified in the experimental groups, among which the most abundant were Bacteroides, Lactobacillus, Phascolarctobacterium, and Akkermansia (see Figures 6(a) and 6(b)).

LEfSe analysis is an analysis method that uses linear discriminant analysis (LDA) to estimate the impact of the abundance of each species on diversity and identify communities or species that show significant differences in sample partitioning. A logarithmic LDA score cutoff of 4.0 was used to identify important taxonomic differences (see Figures $7(\mathrm{a})-7(\mathrm{c}))$.

In summary, compared to the control group, the abundances of Verrucomicrobia $(P=0.043)$, Akkermansia $(P=0.043), \quad$ Lactobacillus $(P=0.035)$, and Prevotella $(P=0.0001)$ were significantly decreased in the model group. In addition, the abundances of Bacteroides $(P=0)$, Phascolarctobacterium $\quad(P=0.0002), \quad$ Parabacteroides $(P=0.0003)$, and Clostridium XlVa $(P=0.011)$ were significantly increased in the model group compared with the control group. Furthermore, the relative abundances of Verrucomicrobia in the control group, model group, S. boulardii group, and QBD treatment group were $5.30 \%$, 

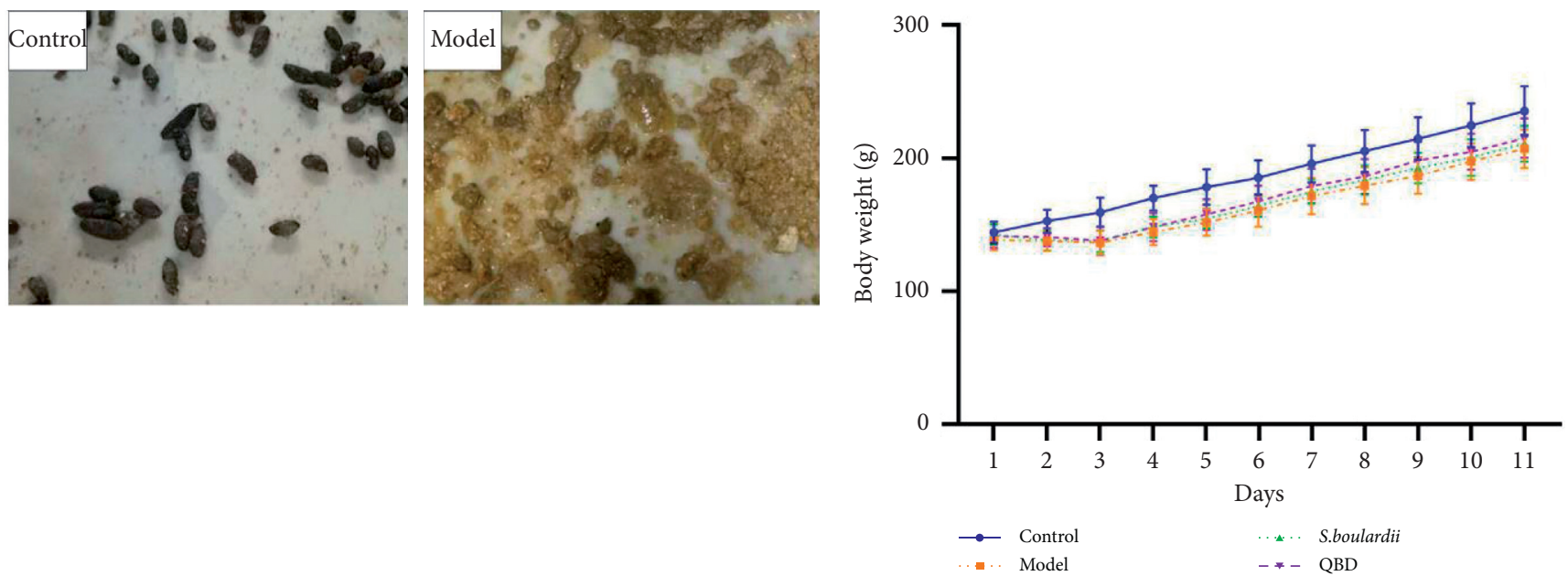

(a)

(b)

FIgURE 1: Efficacy of QBD in alleviating diarrhea. (a) State of feces. (b) Mean weight loss in the experimental groups.

TABLE 1: Colonic mucus thickness (mean $\pm \mathrm{SD}, n=39$ ).

\begin{tabular}{lc}
\hline Group & Thickness of the mucus layer $(\mathrm{mm})$ \\
\hline Control group & $0.028 \pm 0.008$ \\
Model group & $0.017 \pm 0.003^{\# \#}$ \\
$S$. boulardii group & $0.021 \pm 0.005^{\#}$ \\
QBD group & $0.022 \pm 0.006$ \\
$F$ & 4.68 \\
$P$ & 0.006 \\
${ }^{\#} P<0.05$ and ${ }^{\# \#} P<0.01$ vs. control group. SD, standard deviation.
\end{tabular}

$2.04 \%, 6.71 \%$, and $7.43 \%$, respectively. Moreover, the relative abundances of Akkermansia accounted for $11.12 \%, 2.62 \%$, $7.99 \%$, and $8.70 \%$ in the control group, model group, $S$. boulardii group, and QBD treatment group, and QBD is higher than $S$. boulardii treatment. Diarrhea reduced the relative abundances of Verrucomicrobia and Akkermansia. Although the relative abundances of Verrucomicrobia and Akkermansia among the three groups did not reach statistical significance, the S. boulardii and QBD treatments enhanced the increasing tendencies of Verrucomicrobia and Akkermansia. Moreover, the abundances of Turicibacter $(P=0.037)$ and Flavonifractor $(P=0.043)$ were significantly increased in the model group compared with the QBD group. Compared to the model group, the abundance of Parasutterella $(P=0.043)$ was significantly increased in the $S$. boulardii group.

4.7. Spearman Correlation Analysis of the Gut Microbiota and SCFAs. The different bacterial genera produced by the $16 \mathrm{~S}$ rDNA amplicon sequencing analysis and the SCFA concentration were determined by Spearman correlation analysis. The results were as follows. There was no correlation between acetic acid and any of the different bacterial genera $(P>0.05)$. Propionic acid was positively correlated with Bacteroides, Parabacteroides, and Phascolarctobacterium but negatively correlated with Prevotella and Lactobacillus. Butyric acid was positively correlated with
Prevotella and negatively correlated with Clostridium XlVa. Caproic acid was positively correlated with Prevotella and Lactobacillus and negatively correlated with Bacteroides, Phascolarctobacterium, and Flavonifractor (see Figure 8 and Supplementary Table 2).

\section{Discussion}

The balance of the gut microbiota is closely related to diarrhea. Imbalance between diarrhea and the gut microbiota is a result of mutual cause and effect. The diversity of the gut microbiota of healthy people is higher than that of patients with diarrhea under normal conditions [46]. Although the therapeutic effect of QBD on diarrhea has been confirmed in clinical research, the general mechanism remains unclear. Based on these observations, in this study, 16S rDNA highthroughput sequencing was used to explore the regulatory effect of QBD on the gut microbiota in diarrheal juvenile rats.

In this study, through analysis of sequencing data, a significant difference in the species diversity and structures of the gut microbiota in rats was found between the control group and the model group, while the $S$. boulardii and QBD treatments did not significantly change the species diversity and structures of the gut microbiota, which was consistent with the study of Long et al. [27]. These results may be due to the continuous effect of PEG treatment via drinking water; the S. boulardii and QBD treatments once daily were not enough to reverse the changes in the species diversity and structures of the gut microbiota caused by PEG 3350.

Analysis of the differences in the intestinal flora showed the following:

(1) Compared with the control group, the abundances of Bacteroides, Parabacteroides, Phascolarctobacterium, and Clostridium XIVa were significantly increased in the model group, and the abundances of Verrucomicrobia, Lactobacillus, Akkermansia, and Prevotella were significantly reduced in the model group. 

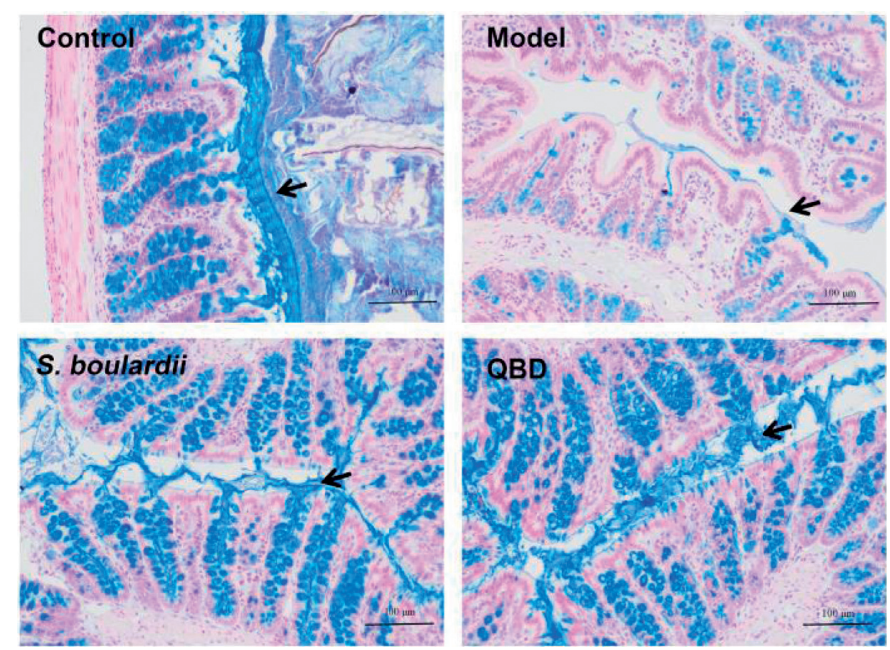

(a)
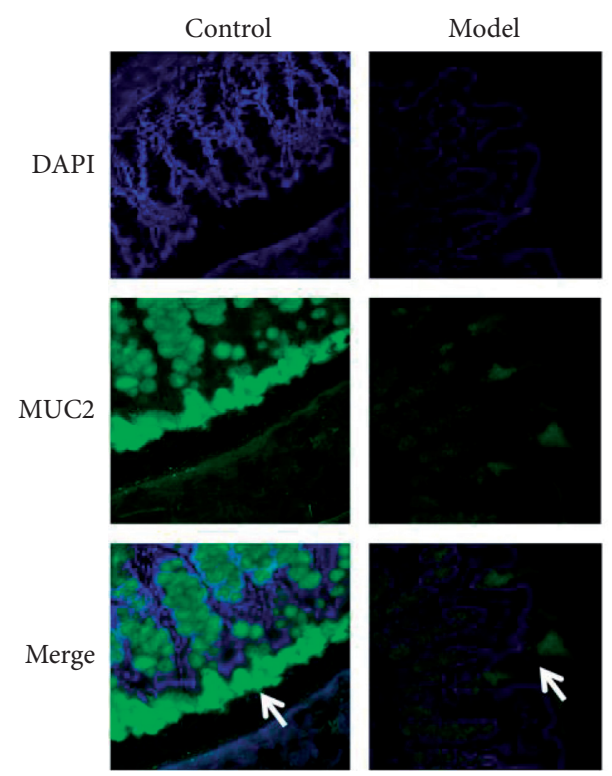

(c)

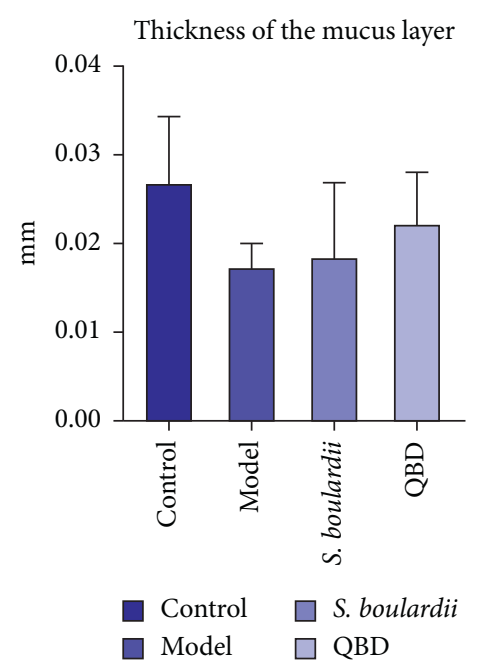

(b)

S. boulardii
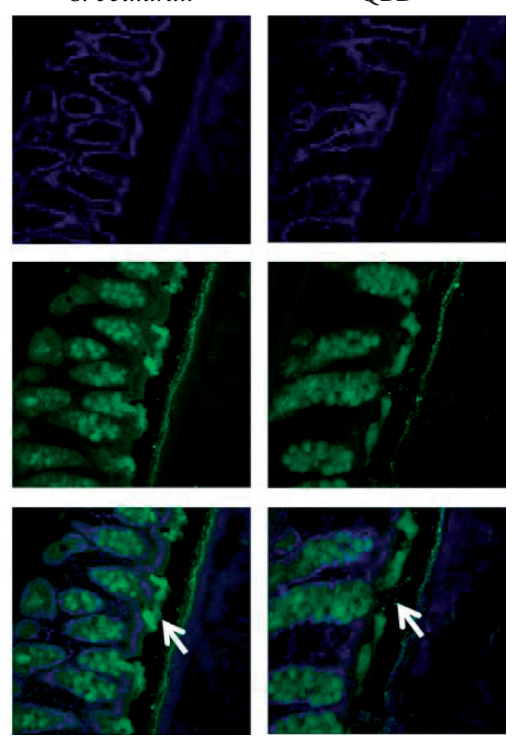

FIGURE 2: QBD alleviated colon mucus layer injury in diarrheal rats after treatment. (a) Alcian blue-stained colonic sections showing the mucus layer. The black arrow indicates the mucus layer. Scale bars, $100 \mu \mathrm{m}$. (b) Colonic mucus layer measurements from Alcian blue-stained sections. Data are presented as the means, and error bars represent the SDs. (c) Expression of MUC2 proteins. White arrows indicate MUC2 proteins. ${ }^{\#} P<0.05$ and ${ }^{\# \#} P<0.01$ vs. the control group.

Specifically, Clostridium XIVa is closely related to protein fermentation and an increased risk of diarrhea [47]. Pop et al. [48] also observed that the abundance of Prevotella in children with diarrhea in low-income countries was decreased. Moreover, the abundance of Akkermansia in the feces of pigs suffering from epidemic diarrhea was significantly decreased. The abundance of Lactobacillus in patients with diarrheal irritable bowel syndrome was significantly decreased, and Lactobacillus treatment significantly improved the symptoms [49]. In addition, the abundances of Lactobacillus, Prevotella, Akkermansia, and Verrucomicrobia in the feces of E. coli O101 diarrhea model rats were significantly reduced. Our results are consistent with these findings.

In summary, Clostridium XIVa may have the effect of exacerbating diarrhea, while Verrucomicrobia, Lactobacillus, Akkermansia, and Prevotella may be beneficial bacteria that have a protective effect against diarrhea; therefore, the imbalance between the increased abundance of Clostridium XIVa and decreased abundance of Verrucomicrobia, Lactobacillus, Akkermansia, and Prevotella may be related to the mechanism underlying diarrhea.

The concentrations of total SCFAs, acetic acid, and propionic acid were increased, and the propionic acid concentration reached statistical significance in 


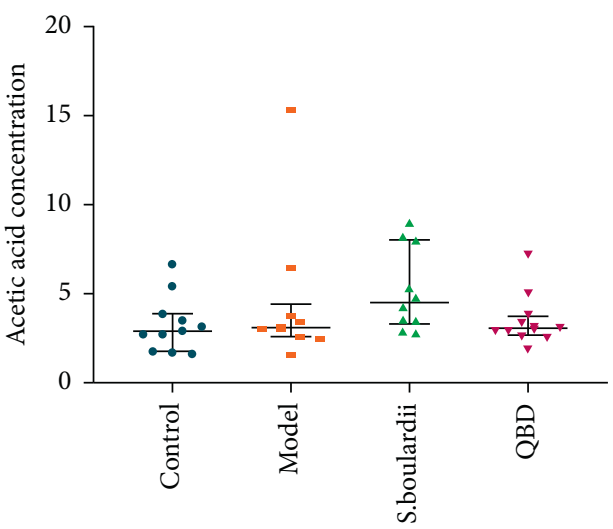

(a)

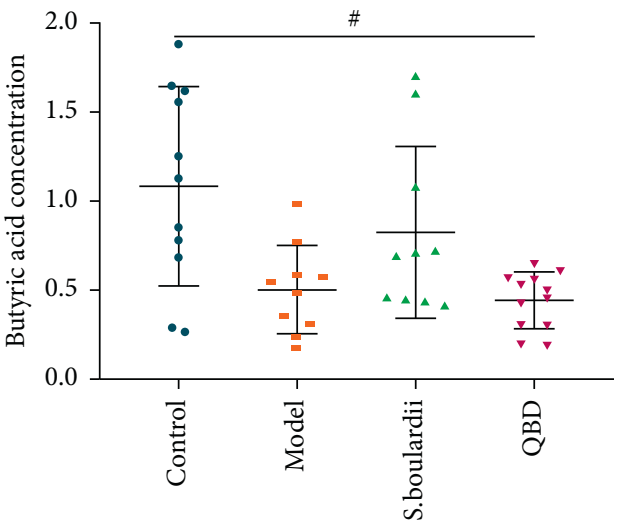

(c)

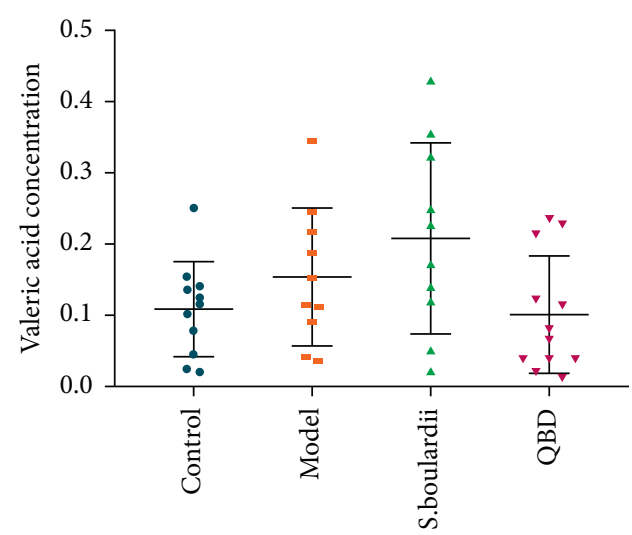

(e)

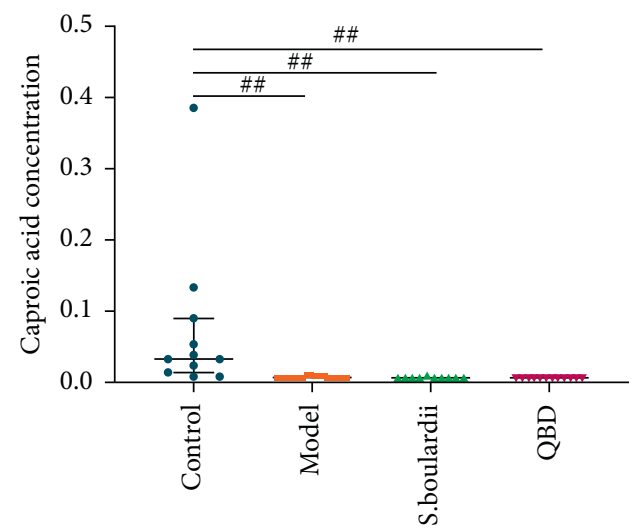

(g)

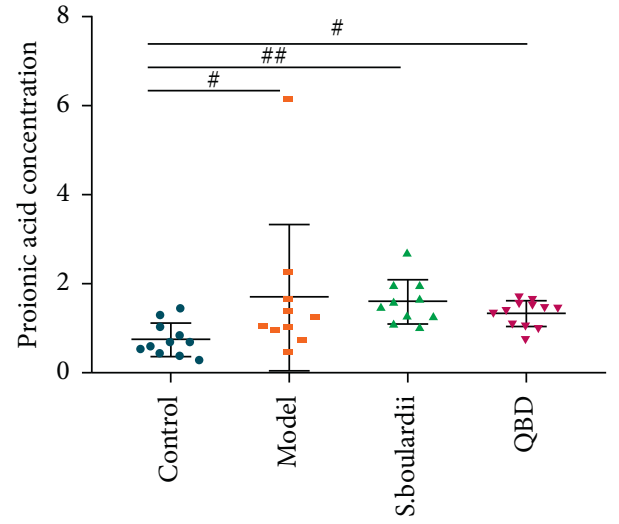

(b)

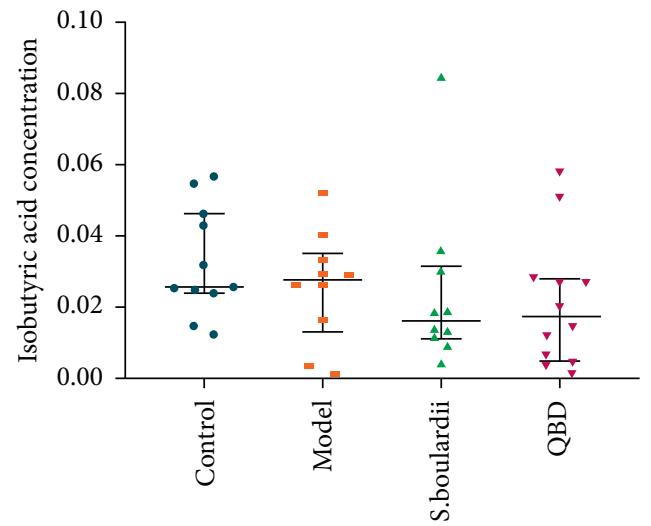

(d)

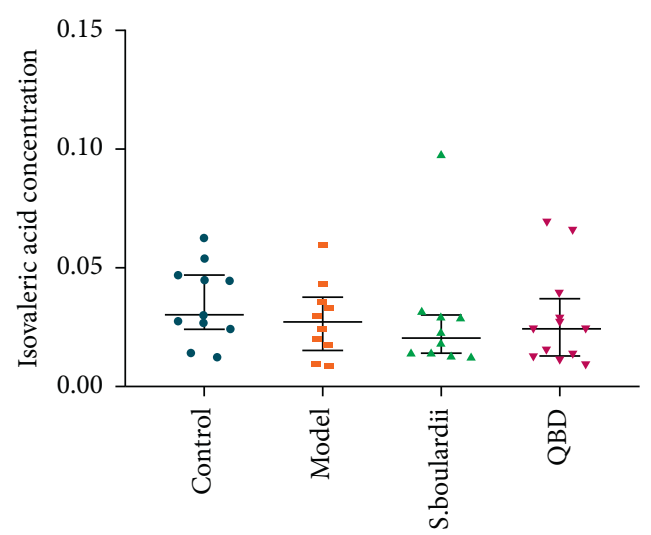

(f)

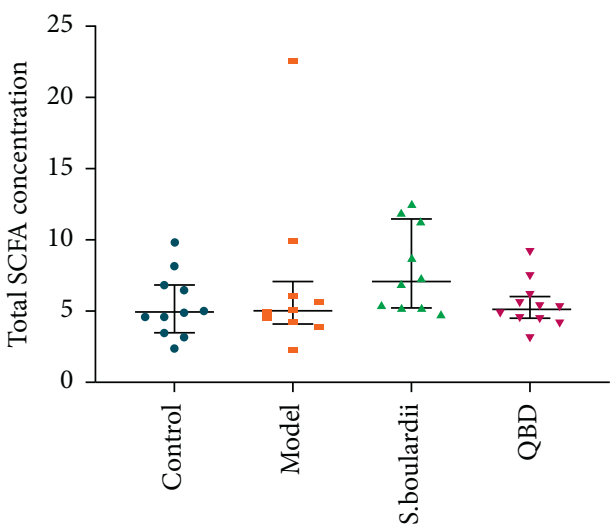

(h)

Figure 3: Concentrations of SCFAs in the colon of each group during the treatment. (a) Acetic acid. (b) Propionic acid. (c) Butyric acid. (d) Isobutyric acid. (e) Valeric acid. (f) Isovaleric acid. (g) Caproic acid. (h) Total short-chain fatty acids. ${ }^{\#} P<0.05$ and ${ }^{\# \#} P<0.01$ vs. control group. 


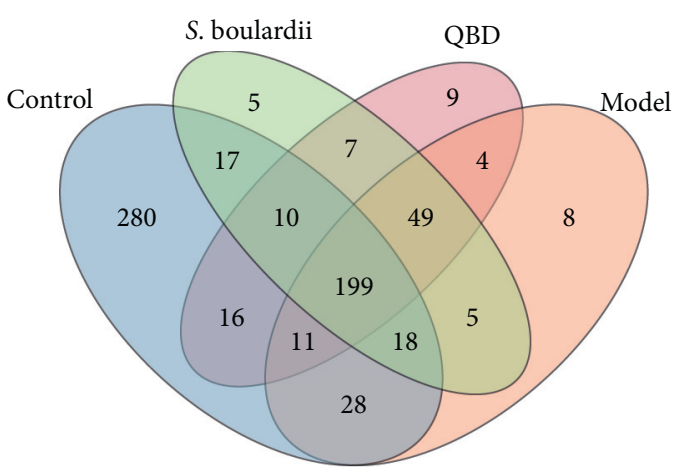

(a)

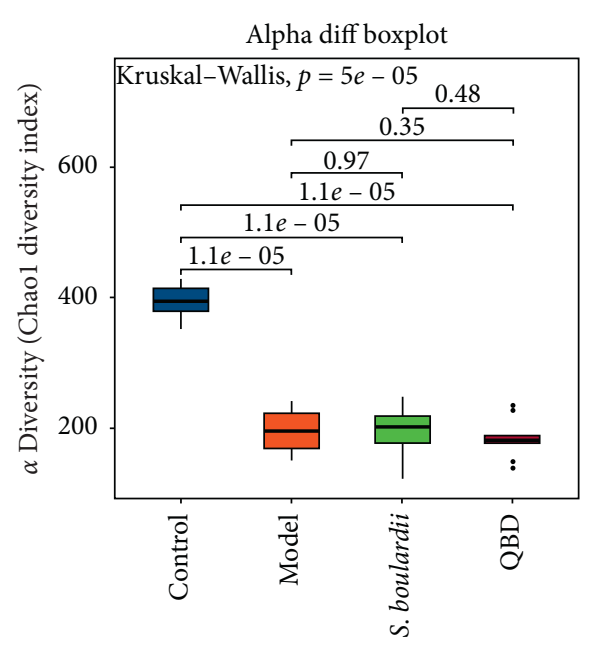

(c)

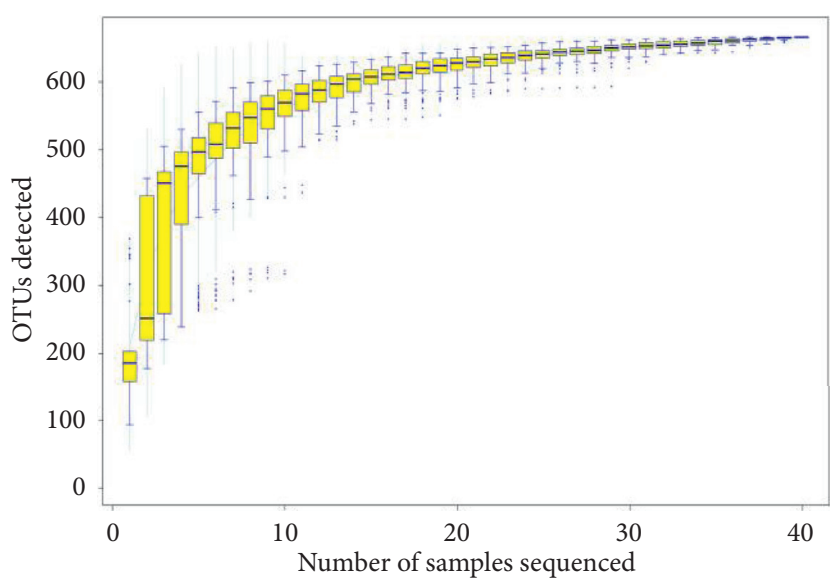

(b)

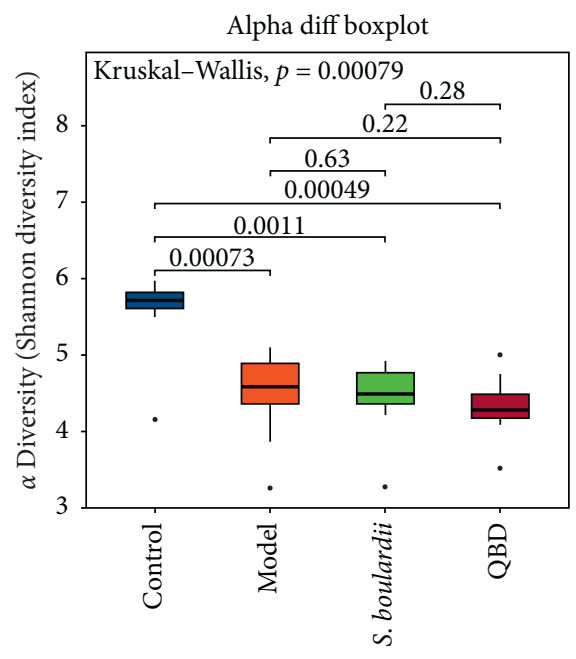

(d)

FIGURE 4: Overall structural modulation of the gut microbiome during QBD treatment. (a) Venn diagram indicating the differential numbers of operational taxonomic units (OTUs) in the control, model, S. boulardii, and QBD groups. (b) Species accumulation curves showing an adequate sampling depth for all samples. (c, d) Alpha diversity analysis of the gut microbiota in the experimental groups based on the Chaol and Shannon indices. $e$ indicates the natural logarithm.

the model group compared to the control group. These findings are similar to those of Whelan et al., who found that the concentrations of fecal total SCFAs, acetic acid, and butyrate increased in patients with diarrhea who were receiving enteral feeding [50]. Moreover, Li et al. also observed that the concentration of propionic acid in the ascending colon of young pigs with rotavirus diarrhea was increased [51]. In this experiment, the abundances of propionic acid-producing bacteria, Bacteroides, Parabacteroides, and Phascolarctobacterium in the three groups using PEG 3350 were significantly increased. The results of Spearman's correlation analysis also confirmed that Bacteroides, Parabacteroides, and Phascolarctobacterium were positively correlated with propionic acid. This may be the reason for the increased concentration of propionic acid in diarrhea samples. A mechanistic explanation may be that the faster transit time in the small intestine during diarrhea leads to malabsorption of carbohydrates over this short period of time, which increases the level of substrate for colonic fermentation, promoting decomposition by these flora, and leading to increased SCFA production [52, 53].

(2) QBD treatment enhanced the increasing tendencies of the abundances of Verrucomicrobia and Akkermansia, whereas those of Turicibacter and Flavonifractor were significantly reduced, compared to the model group. To date, there have been few studies on Turicibacter and Flavonifractor, which are mostly negatively related to health [54-56]. Importantly, accumulating studies have indicated that Verrucomicrobia and Akkermansia are closely related to diarrhea and the intestinal mucus barrier. Therefore, we hypothesized that QBD treatment modulates the gut microbiota and repairs the mucus barrier in juvenile diarrheal rats by the following mechanism: (1) QBD treatment prompts an increase in 

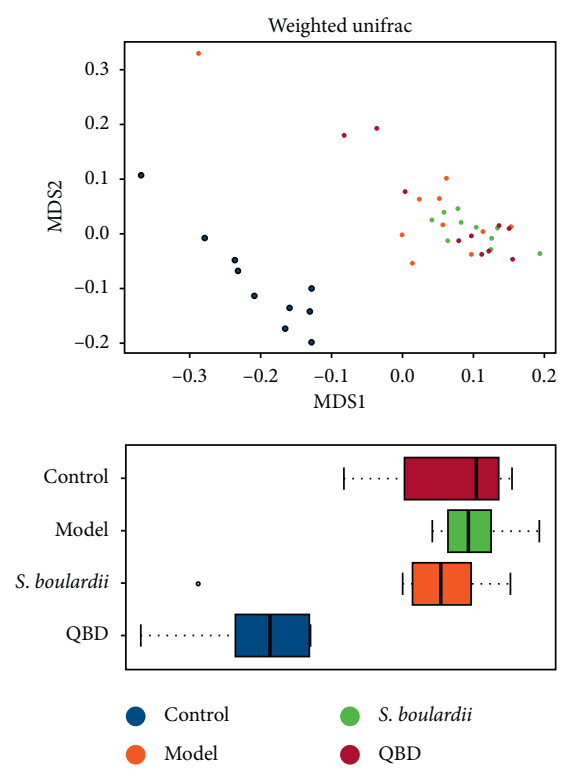

(a)
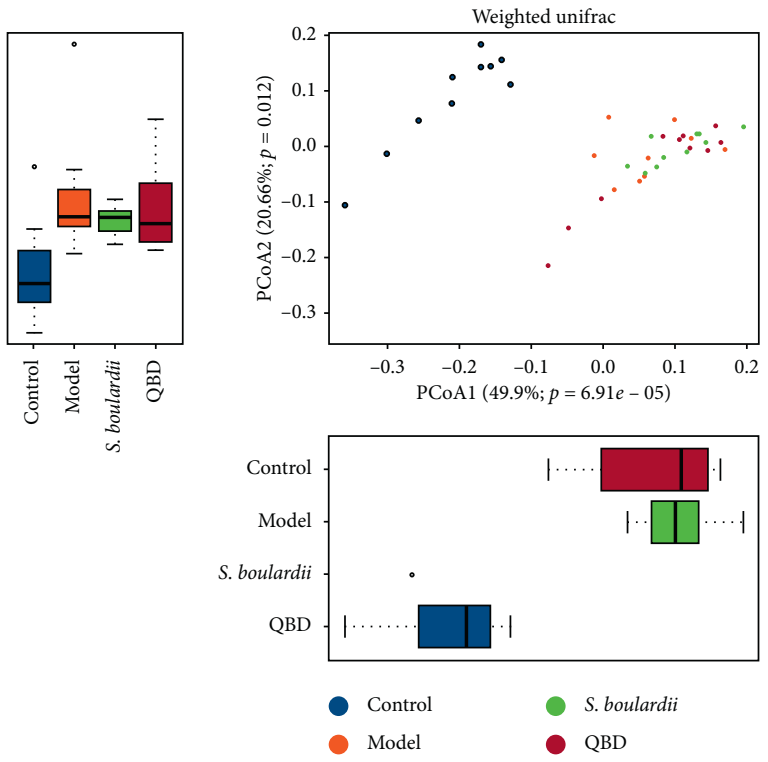

(b)

FIGURE 5: (a) Nonmetric multidimensional scaling (NMDS) and (b) principal coordinate analysis (PCoA) scores based on weighted Unifrac metrics indicating different beta diversity of gut microbiota among the experimental groups.

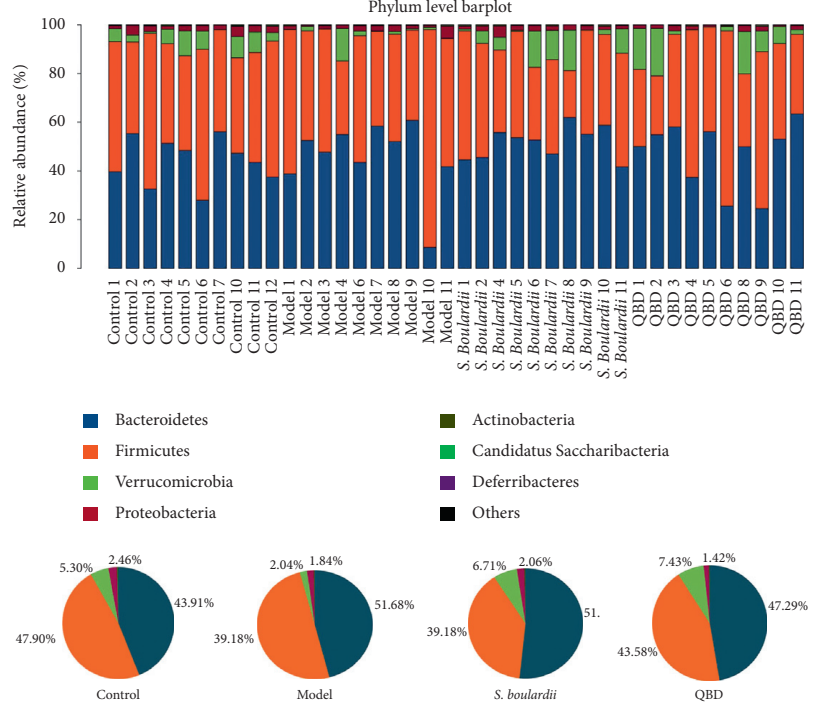

(a)

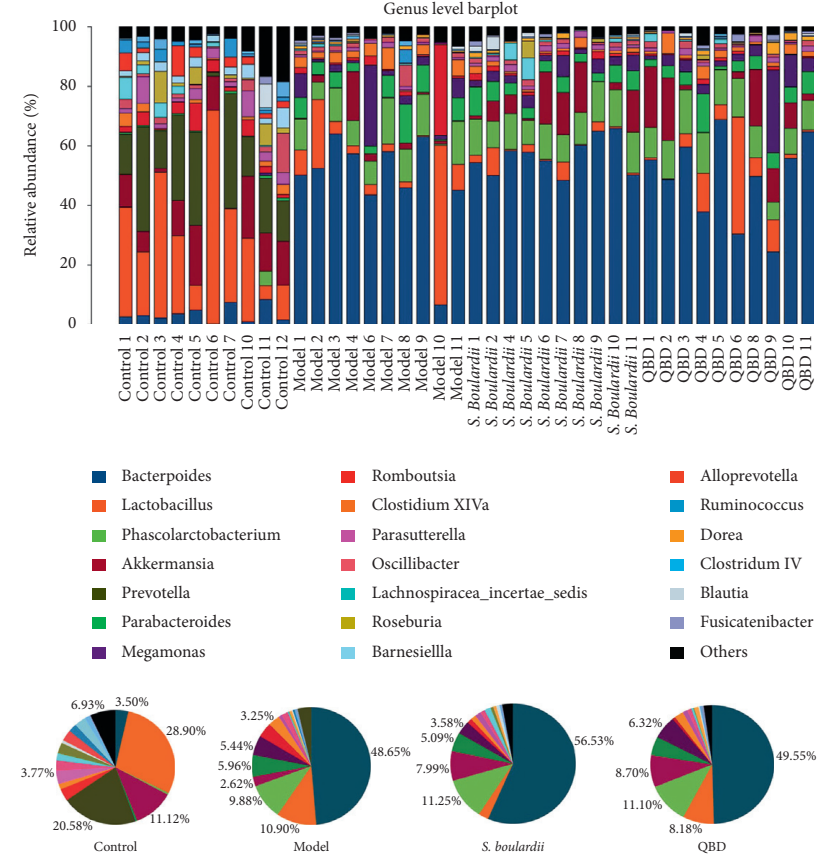

(b)

FIgURE 6: (a) The histogram shows the relative abundance at the phylum level in the 40 samples, and the pie charts show the corresponding microbiota community structures in the experimental group. (b) The histogram shows the relative abundance at the genus level in the 40 samples, and the pie charts show the corresponding microbiota community structures in the experimental group.

Verrucomicrobia abundance and thereby improves the intestinal barrier [57]. (2) QBD treatment increases the abundance of Akkermansia, the most representative bacteria in the phylum Verrucomicrobia. Akkermansia and mucin glycoside hydrolase expression help maintain the thickness of the 


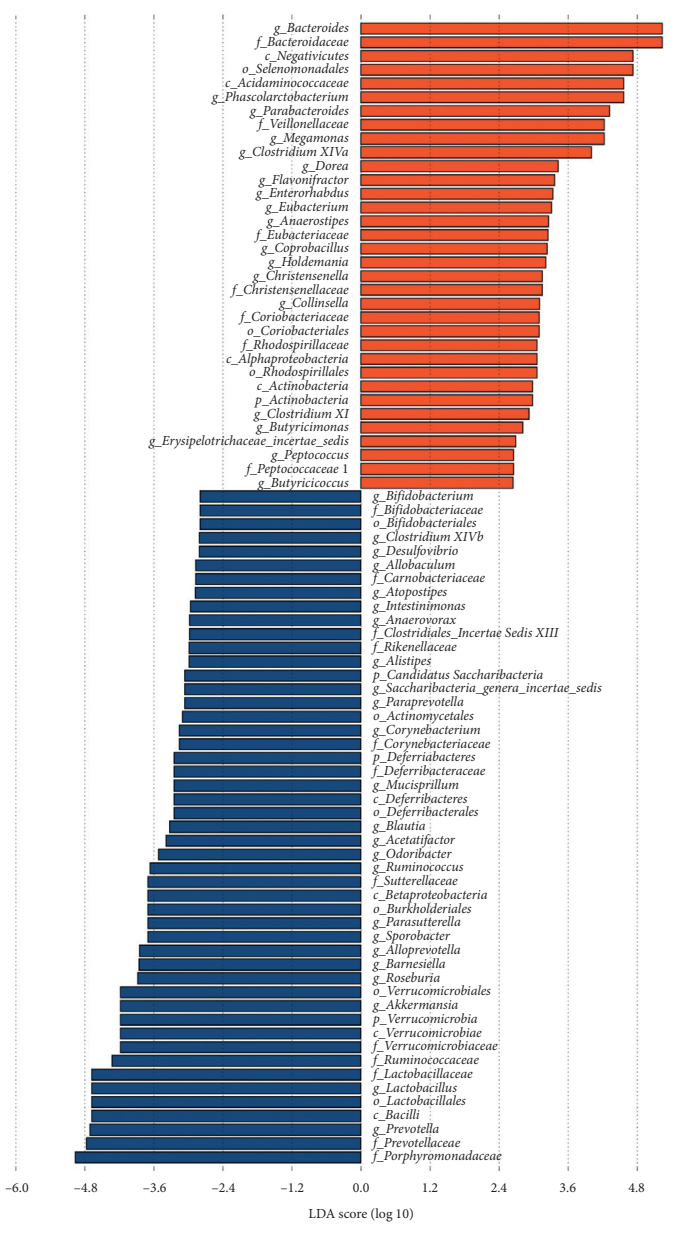

ש Control

(a)

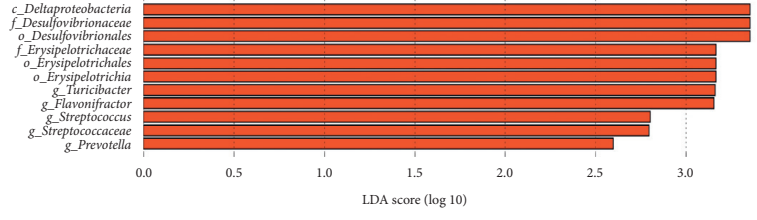

a Model

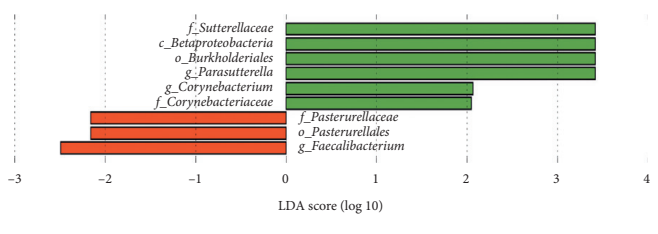

Model
․ boulardi

(c)

FIGURE 7: (a) LEfSE comparison of gut microbiota between the control and model groups. (b) LEfSE comparison of gut microbiota between the model and QBD groups. (c) LEfSE comparison of gut microbiota between the model and S. boulardii groups.

mucosal layer during PEG-induced diarrhea, which suggests a microbiota-driven feedback mechanism aiding mucus resilience [3].

Importantly, the mucus barrier and its most important protein, MUC2, protect the intestines and prevent diarrhea. Xu et al. found that MUC2 is an important protein for the prevention and treatment of rotavirus infection and diarrhea, acting by protecting the epithelial barrier and resisting intestinal permeability [58]. Wang et al. also observed that increasing the content of MUC2 in the ileum of rats with diarrhea can enhance intestinal barrier defense and prevent diarrhea [59]. Interestingly, TCM herbs (fermented Rhizoma Atractylodis Macrocephalae) increase the proportion of Akkermansia and simultaneously have a beneficial effect on host metabolism [60]. Zhang et al. also found that Sijunzi decoction contained in QBD increased the expression of MUC2 and improved the state of spleen deficiency in rats [61]. Sun et al. previously showed that QBD regulated the imbalance of the gut microbiota and promoted the repair of intestinal mucosal damage in diarrheal mice [62]. Our results are consistent with this finding. 


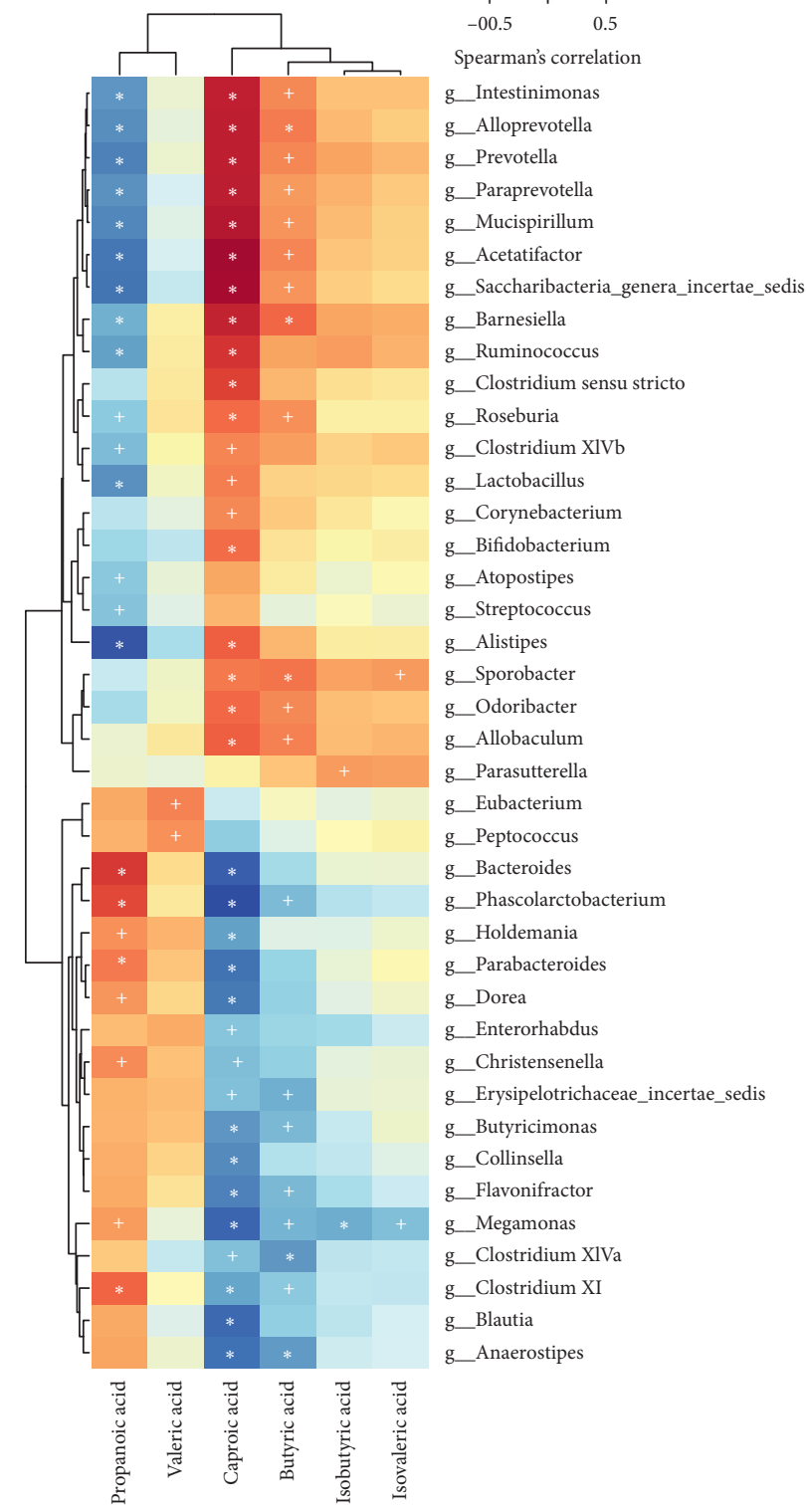

Figure 8: Heat map of the Spearman correlation analysis between the gut microbiota and SCFAs. ${ }^{+} P<0.05 ;{ }^{*} P<0.01$.

In summary, based on the results showing that the mucus layer thickness and MUC2 expression in the QBD group were greater than those in the model group, it is suggested that $\mathrm{QBD}$ may regulate the intestinal flora, such as by increasing the abundances of Verrucomicrobia and Akkermansia, to repair the mucus barrier, protect the intestines, and treat diarrhea.

(3) The abundance of Parasutterella in the S. boulardii group was significantly higher than that in the model group. Chen et al. observed that pectin increased the abundances of Bacteroides and Akkermansia and significantly increased the abundances of Parasutterella, which may have contributed to increasing the concentrations of acetic acid, propionic acid, and butyric acid in the cecum [63]. Li et al. reported that reductions in the abundance of Lactobacillus,
Parasutterella, and Desulfovibrio and the levels of acetic acid, propionic acid, and butyric acid were consistent after azithromycin treatment in mice [64]. The results of these two studies are consistent with our findings regarding the change trends of some gut microbes and SCFAs, suggesting that the abundance of Parasutterella is positively correlated with the levels of acetic acid, propionic acid, and butyric acid.

The GC-MS results showed that although the difference was not statistically significant, the total levels of SCFAs, acetic acid, propionic acid, and butyric acid in the $S$. boulardii group were higher than those in the model group. Pipau et al. found that $S$. boulardii alleviated diarrhea by increasing the concentrations of acetic acid, propionic acid, and butyric acid [65].

Based on these findings, we speculate that $S$. boulardii can increase the abundance of Parasutterella, resulting in an 


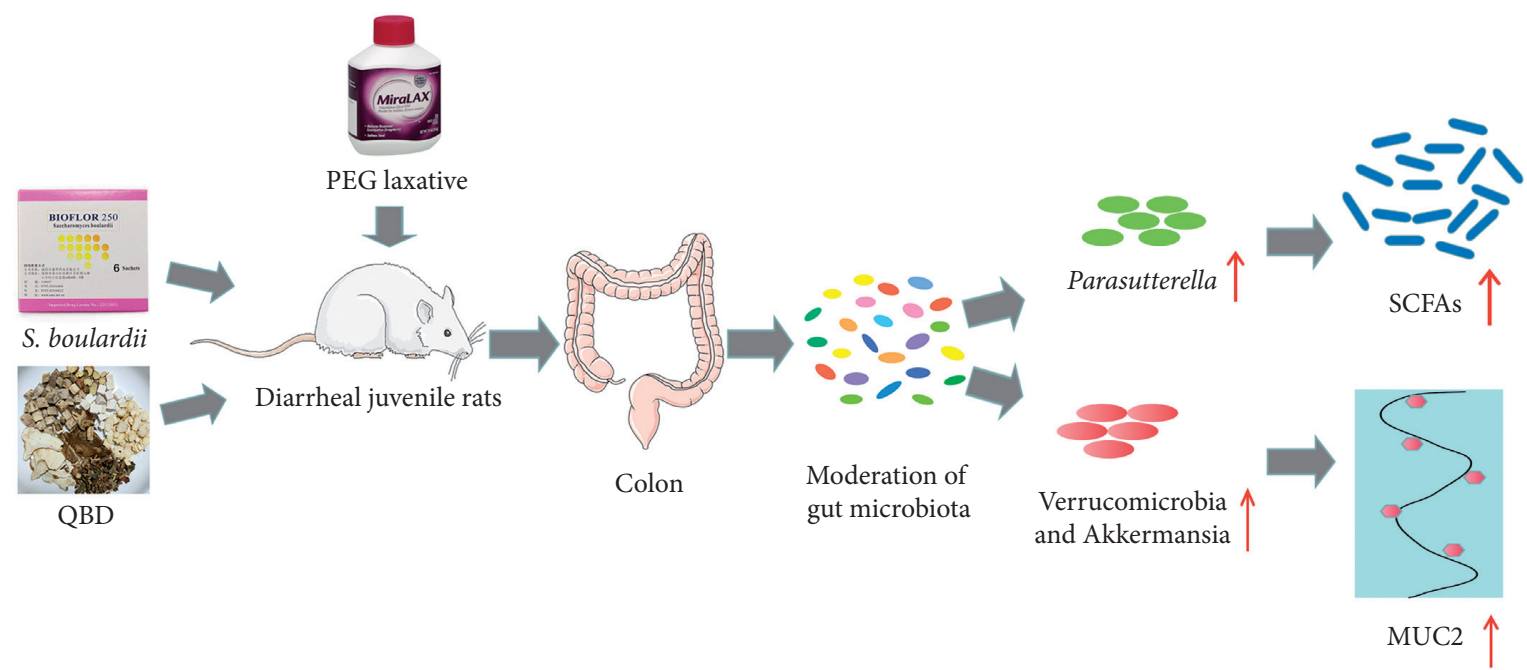

FIgURE 9: Possible mechanism underlining S. boulardii and QBD treatment of diarrhea in juvenile rats (note: the rat and colon images are from https://smart.servier.com/).

increase in the levels of acetic acid, propionic acid, and butyric acid, consequently leading to alleviation of diarrhea (see Figure 9).

\section{Conclusion}

QBD has a tendency to improve the weight gain of juvenile rats with diarrhea. QBD has a stronger ability to repair the mucus barrier than $S$. boulardii, but its ability to improve SCFAs is not as good as that of S. boulardii. It is suggested that $S$. boulardii may be able to treat diarrhea by increasing the abundance of Parasutterella significantly to increase SCFA production, and the mechanism of action of QBD in the treatment of juvenile rats with diarrhea likely involves gut microbiota-mediated repair of the mucus barrier.

\section{Data Availability}

The data used to support the findings of this study are available from the corresponding author upon request.

\section{Conflicts of Interest}

All the authors declare that they have no conflicts of interest.

\section{Authors' Contributions}

Fei Luo and Xiaogang Chen designed the study. Yang Yang, Jingtu Cen, Peiwen Chen, Liyan Ye, Liying Zeng, Qina Ye, Xiangna Yang, and Jiayi Shan performed the experiment and collected the data. Shaodan Sun and Xiaojie Lin analyzed the data. Shaodan Sun wrote the manuscript. Li Xie and Meirong Jiang reviewed the manuscript.

\section{Acknowledgments}

This study was supported in part by the National Science Foundation of China (Nos. 81173303 and 81674021) and the Natural Science Foundation of Guangdong Province (2017A030310073).

\section{Supplementary Materials}

Supplementary Table 1: the active ingredients of the seven components of QBD. Supplementary Table 2: Spearman correlation analysis between the gut microbiota and SCFAs. (Supplementary Materials)

\section{References}

[1] M. Naghavi, A. A. Abajobir, C. Abbafati et al., "Global, regional, and national age-sex specific mortality for 264 causes of death, 1980-2016: a systematic analysis for the Global Burden of Disease Study 2016," The Lancet, vol. 390, no. 10100, pp. 1151-1210, 2017.

[2] C. Troeger, B. F. Blacker, I. A. Khalil et al., "Estimates of the global, regional, and national morbidity, mortality, and aetiologies of diarrhoea in 195 countries: a systematic analysis for the Global Burden of Disease Study," The Lancet Infectious Diseases, vol. 18, no. 11, pp. 1211-1228, 2016.

[3] C. Tropini, E. L. Moss, B. D. Merrill et al., "Transient osmotic perturbation causes long-term alteration to the gut microbiota," Cell, vol. 173, no. 7, pp. 1742-1754, 2018.

[4] G. Gorkiewicz, G. G. Thallinger, S. Trajanoski et al., "Alterations in the colonic microbiota in response to osmotic diarrhea," PLoS One, vol. 8, no. 2, Article ID e55817, 2013.

[5] H. Chung The, P. F. de Sessions, S. Jie et al., "Assessing gut microbiota perturbations during the early phase of infectious diarrhea in Vietnamese children," Gut Microbes, vol. 9, no. 1, pp. 38-54, 2018.

[6] M. Pop, A. W. Walker, J. Paulson et al., "Diarrhea in young children from low-income countries leads to large-scale alterations in intestinal microbiota composition," Genome Biology, vol. 15, no. 6, p. R76, 2014.

[7] R. H. J. Bandsma, K. Sadiq, and Z. A. Bhutta, "Persistent diarrhoea: current knowledge and novel concepts," Paediatrics and International Child Health, vol. 39, no. 1, pp. 41-47, 2018.

[8] S. Rouhani, N. W. Griffin, P. P. Yori et al., "Diarrhea as a potential cause and consequence of reduced gut microbial diversity among undernourished children in Peru," Clinical Infectious Diseases, vol. 71, no. 4, pp. 989-999, 2020. 
[9] J. H. Cummings, E. W. Pomare, W. J. Branch, C. P. Naylor, and G. T. Macfarlane, "Short chain fatty acids in human large intestine, portal, hepatic and venous blood," Gut, vol. 28, no. 10, pp. 1221-1227, 1987.

[10] J. Plaza-Diaz, F. J. Ruiz-Ojeda, M. Gil-Campos, and A. Gil, "Mechanisms of action of probiotics," Advances in Nutrition, vol. 10, no. 1, pp. S49-S66, 2019.

[11] H. J. Binder, "Role of colonic short-chain fatty acid transport in diarrhea," Annual Review of Physiology, vol. 72, pp. 297-313, 2010.

[12] O. C. Velázquez, H. M. Lederer, and J. L. Rombeau, "Butyrate and the colonocyte. Production, absorption, metabolism, and therapeutic implications," Advances in Experimental Medicine and Biology, vol. 427, pp. 123-134, 1997.

[13] M. E. Johansson, H. Sjövall, and G. C. Hansson, "The gastrointestinal mucus system in health and disease," Nature Reviews Gastroenterology \& Hepatology, vol. 10, no. 6, pp. 352-361, 2013.

[14] M. Wlodarska and B. B. Finlay, "Host immune response to antibiotic perturbation of the microbiota," Mucosal Immunology, vol. 3, no. 2, pp. 100-103, 2009.

[15] A. Guarino, S. Guandalini, and A. Lo Vecchio, "Probiotics for prevention and treatment of diarrhea," Journal of Clinical Gastroenterology, vol. 49, no. 1, pp. S37-S45, 2015.

[16] S. Asmat, F. Shaukat, R. Asmat, H. Bakhat, and T. M. Asmat, "Clinical efficacy comparison of Saccharomyces boulardii and lactic acid as probiotics in acute pediatric diarrhea," Journal of College of Physicians And Surgeons Pakistan, vol. 28, no. 3, pp. 214-217, 2018.

[17] P. Periti and F. Tonelli, "Preclinical and clinical pharmacology of biotherapeutic agents: Saccharomyces boulardii," Journal of Chemotherapy, vol. 13, no. 5, pp. 473-493, 2001.

[18] L. V. McFarland and P. Bernasconi, "Saccharomyces boulardii'. A review of an innovative biotherapeutic agent," Microbial Ecology in Health and Disease, vol. 6, no. 4, pp. 157-171, 1993.

[19] R. Zbinden, "Inhibition of Saccharomyces boulardii (nom. inval.) on cell invasion of Salmonella typhimurium and Yersinia enterocolitica," Microbial Ecology in Health and Disease, vol. 11, no. 3, pp. 158-162, 2009.

[20] D. Czerucka, S. Dahan, B. Mograbi, B. Rossi, and P. Rampal, "Saccharomyces boulardii preserves the barrier function and modulates the signal transduction pathway induced in enteropathogenic Escherichia coli-infected T84 cells," Infection and Immunity, vol. 68, no. 10, pp. 5998-6004, 2000.

[21] P. Girard, Y. Pansart, I. Lorette, and J. M. Gillardin, "Doseresponse relationship and mechanism of action of Saccharomyces boulardii in castor oil-induced diarrhea in rats," Digestive Diseases and Sciences, vol. 48, no. 4, pp. 770-774, 2003.

[22] M. F. Geyik, M. Aldemir, S. Hosoglu et al., "The effects of Saccharomyces boulardii on bacterial translocation in rats with obstructive jaundice," Annals of the Royal College of Surgeons of England, vol. 88, no. 2, pp. 176-180, 2006.

[23] A. Sezer, U. Usta, and I. Cicin, "The effect of Saccharomyces boulardii on reducing irinotecan-induced intestinal mucositis and diarrhea," Medical Oncology, vol. 26, no. 3, pp. 350-357, 2009.

[24] L. Wang, G. B. Zhou, P. Liu et al., "Dissection of mechanisms of Chinese medicinal formula Realgar-Indigo naturalis as an effective treatment for promyelocytic leukemia," Proceedings of the National Academy of Sciences of the United States of America, vol. 105, no. 12, pp. 4826-4831, 2008.
[25] H. Hui, Y. Wu, T. Zheng, S. Zhou, and Z. Tan, "Bacterial characteristics in intestinal contents of antibiotic-associated diarrhea mice treated with Qiweibaizhu powder," Medical Science Monitor, vol. 26, Article ID e921771, 2020.

[26] C. Long, Y. Liu, L. He et al., "Bacterial lactase genes diversity in intestinal mucosa of dysbacterial diarrhea mice treated with Qiweibaizhu powder," 3 Biotech, vol. 8, no. 10, p. 423, 2018.

[27] C. X. Long, H. Q. Shao, C. Y. Luo, R. Yu, and Z. J. Tan, "Bacterial diversity in the intestinal mucosa of dysbiosis diarrhea mice treated with Qiweibaizhu powder," Gastroenterology Research and Practice, vol. 2020, Article ID 9420129, 8 pages, 2020.

[28] X. P. Kangxiao Guo, Y. Mao, S. Xu, Z. Yang, Z. Tan, and S. Zhou, "Effect of Qiwei Baizhu San on intestinal sucrase activity in mice with diarrhea," Chinese Journal of Microecology, vol. 10, p. 3, 2019.

[29] H. Li, Effect of Qiwei Baizhu Powder Combined with Magnetic (Arctic) Sticking Tianshu Point on Intestinal Flora of Spleen DeficiencySyndrome and Diarrhea in Young RatsAbstrac, Hunan University of Chinese Medicine, Changsha, China, 2019.

[30] W. Liu, Different Formulations Qiweibaizhu Powder on Intestinal Flora in Mice with Diarrhea and the Treatment of Immune-Related Mechanism, Hunan University of Chinese Medicine, Changsha, China, 2015.

[31] C. W. Biqiang Sun, Y. Zhou, W. Liu et al., "Effects of different formulations of Qiwei Baizhu Powder on the ultrastructure of small intestinal mucosa and $\operatorname{sg} \mathrm{A}$ in mice with intestinal dysbacteriosis," Chinese Journal of Microecology, vol. 28, no. 2, pp. 125-128, 2016.

[32] Y. Z. Biqiang Sun, W. Liu, and C. Wu, "Effects of different formulated Qiwei Baizhu powder on intestinal flora and tight junction protein in diarrhea mice," Lishizhen Medicine And Materia Medica Research, vol. 26, no. 12, pp. 2835-2837, 2015.

[33] Y. Z. Biqiang Sun, W. Liu, and C. Wu, "Effects of different formulated Qiwei Baizhu san on intestinal mucosa and TNF$\alpha$, IL-6 in serum of mice with intestinal flora," Chinese Journal of Experimental Traditional Medical Formulae, vol. 22, no. 4, pp. 95-100, 2016.

[34] Z. Ting, C. Guangxian, W. Hai, W. Dan, and T. Zhoujin, "Impacts of plant components to intestinal tract microflora," China Pharmaceuticals, vol. 18, pp. 19-21, 2010.

[35] C. Jun-min, Y. Xue-jing, and Z. Wei-zhen, "Study on the effect and mechanism of Chinese herbs on intestinal normal flora of mice," Chinese Archives of Traditional Chinese Medicine, vol. 30, no. 2, pp. 393-395, 2012.

[36] J. Jie, G. Kang-xiao, L. Ling, L. Dan-dan, T. Zhou-jin, and Y. Zhen-yi, "Inhibiting effect of ultra-micro powder Qiweibaizhusan on bacteria in vitro," Chinese Journal of Information on TCM, vol. 20, no. 11, pp. 28-30, 2013.

[37] J. Baolin, "Study about the regulation of decoction of four noble drugson intestinal microbiology dysbiosis and its mechanism," Jouranal of Mudanjiang Medical College, vol. 28, no. 5, pp. 20-23, 2007.

[38] L. Li-sha, W. Rui, X. Ri-hua et al., "Promoting effect of polysaccharides isolated from rhizoma Atractylodis Macrocephalae on the growth of probiotics and structure analysis," Food and Science, vol. 19, pp. 124-128, 2010.

[39] T. Zhoujin, W. Hai, L. Fulin et al., "Effect of ultra-micro powder qiweibaishusan on the intestinal microbiota and enzyme activities in mice," Acta Ecologica Sinica, vol. 32, no. 21, pp. 6856-6863, 2012.

[40] Z. Ji-zhen, Z. Wenju, M. Qing, and LuoJianping, "Research advance on pharmacological activity and content 
accumulation of chemical component from Ginseng," Journal of Anhui Agriculture, vol. 39, no. 20, pp. 12158-12160, 2011.

[41] Z. Ao, Z. Hua-Ling, T. Zhou-Jin, C. Ying, C. Guang-Xian, and Z. Sai-Nan, "The construction of mice diarrhea model due to dysbacteriosis and curative effect of ultra-micro Qiweibaizhusan," Microbiology China,, vol. 39, no. 9, pp. 1341-1348, 2012.

[42] X. Xu, W. Zhang, C. Huang et al., "A novel chemometric method for the prediction of human oral bioavailability," International Journal of Molecular Sciences, vol. 13, no. 6, pp. 6964-6982, 2012.

[43] W. Zhang, B. Zhu, J. Xu et al., "Bacteroides fragilis protects against antibiotic-associated diarrhea in rats by modulating intestinal defenses," Frontiers in Immunology, vol. 9, p. 1040, 2018.

[44] K. A. Earle, G. Billings, M. Sigal et al., "Quantitative imaging of gut microbiota spatial organization," Cell Host \& Microbe, vol. 18, no. 4, pp. 478-488, 2015.

[45] M. E. V. Johansson and G. C. Hansson, "Preservation of mucus in histological sections, immunostaining of mucins in fixed tissue, and localization of bacteria with FISH," Methods in Molecular Biology, vol. 842, pp. 229-235, 2012.

[46] X. Sun, Y. Gao, X. Wang et al., "Escherichia coli O(101)induced diarrhea develops gut microbial dysbiosis in rats," Experimental and Therapeutic Medicine, vol. 17, no. 1, pp. 824-834, 2018.

[47] V. T. Rist, E. Weiss, N. Sauer, R. Mosenthin, and M. Eklund, "Effect of dietary protein supply originating from soybean meal or casein on the intestinal microbiota of piglets," $A n$ aerobe, vol. 25, pp. 72-79, 2013.

[48] M. Pop, A. W. Walker, J. Paulson et al., "Diarrhea in young children from low-income countries leads to large-scale alterations in intestinal microbiota composition," Genome Biology, vol. 15, no. 6, p. R76, 2014.

[49] H. N. Liu, H. Wu, Y. Z. Chen, Y. J. Chen, X. Z. Shen, and T. T. Liu, "Altered molecular signature of intestinal microbiota in irritable bowel syndrome patients compared with healthy controls: a systematic review and meta-analysis," Digestive and Liver Disease, vol. 49, no. 4, pp. 331-337, 2017.

[50] K. Whelan, P. A. Judd, K. M. Tuohy, G. R. Gibson, V. R. Preedy, and M. A. Taylor, "Fecal microbiota in patients receiving enteral feeding are highly variable and may be altered in those who develop diarrhea," The American Journal of Clinical Nutrition, vol. 89, no. 1, pp. 240-247, 2009.

[51] M. Li, M. H. Monaco, M. Wang et al., "Human milk oligosaccharides shorten rotavirus-induced diarrhea and modulate piglet mucosal immunity and colonic microbiota," ISME J, vol. 8, no. 8, pp. 1609-1620, 2014.

[52] S. J. Lewis and K. W. Heaton, "Increasing butyrate concentration in the distal colon by accelerating intestinal transit," Gut, vol. 41, no. 2, pp. 245-251, 1997.

[53] J. A. Vogt and T. M. Wolever, "Fecal acetate is inversely related to acetate absorption from the human rectum and distal colon," Journal of Nutrition, vol. 133, no. 10, pp. 3145-3148, 2003.

[54] F. K. Berger, N. Schwab, M. Glanemann, R. M. Bohle, B. Gärtner, and H. V. Groesdonk, "Flavonifractor (Eubacterium) plautii bloodstream infection following acute cholecystitis," IDCases, vol. 14, Article ID e00461, 2018.

[55] T. A. Auchtung, M. E. Holder, J. R. Gesell et al., "Complete genome sequence of turicibacter sp. strain H121, isolated from the feces of a contaminated germ-free mouse," Genome Announc, vol. 4, no. 2, 2016.
[56] P. Cuív, E. S. Klaassens, A. S. Durkin et al., "Draft genome sequence of Turicibacter sanguinis PC909, isolated from human feces," Journal of Bacteriology, vol. 193, no. 5, pp. 1288-1289, 2011.

[57] X. Li, Y. Wu, Z. Xu et al., "Effects of hetiao Jianpi decoction on intestinal injury and repair in rats with antibiotic-associated diarrhea," Medical Science Monitor, vol. 26, Article ID e921745, 2020.

[58] R. Xu, Y. H. Lei, J. Shi, Y. J. Zhou, Y. W. Chen, and Z. J. He, "Effects of lactadherin on plasma D-lactic acid and small intestinal MUC2 and claudin-1 expression levels in rats with rotavirus-induced diarrhea," Experimental and Therapeutic Medicine, vol. 11, no. 3, pp. 943-950, 2016.

[59] W. Yaying, T. Linjie, L. Jjao et al., "Effect of Lactobacills casei on the ileal mucosal structure and MUC2 content of rats with developmental diarrhea," Journal of China Agricultural University, vol. 24, no. 8, pp. 94-101.

[60] M. Derrien, C. Belzer, and W. M. de Vos, "Akkermansia muciniphila and its role in regulating host functions," Microbial Pathogenesis, vol. 106, pp. 171-181, 2017.

[61] Z. Bo, Experimental Study on the Change of Intestinal Mucosal Barrier Function in Spleen Deficiency Rats and the Effect of Sijunzi Decoction on it, Liaoning University of Traditional Chinese Medicine, Shenyang, China, 2014.

[62] S. Bi-qiang, Z. Ying, L. Wei-dong, and W. Can-rong, "Effects of different formulated Qiwei Baizhu powder on intestinal flora and tight junc-tion protein in diarrhea mice," Lishizhen Medicine and Materia Medica Research, vol. 26, no. 12, pp. 2835-2837, 2020.

[63] C. Junliang, Effect of Different Pectin on Gut Microbiota and Selected Physiological Parameters in Obese Mice, Jina University, Guangzhou, China, 2018.

[64] R. Li, H. Wang, Q. Shi et al., "Effects of oral florfenicol and azithromycin on gut microbiota and adipogenesis in mice," PLoS One, vol. 12, no. 7, Article ID e0181690, 2017.

[65] F. Girard-Pipau, A. Pompei, J. Nano, X. Boquet, and P. Rampal, "Intestinal microflora, short chain and cellular fatty acids, influence of a probiotic Saccharomyces boulardii," Microbial Ecology in Health and Disease, vol. 14, no. 4, pp. 221-228, 2009. 